\title{
A combined NMR and DFT study of Narrow Gap Semiconductors: The case of PbTe
}

\author{
Robert E. Taylor ${ }^{1}$, Fahri Alkan ${ }^{2}$, Dimitrios Koumoulis ${ }^{1}$, Michael P. Lake ${ }^{1}$, Daniel King ${ }^{1}$, Cecil \\ Dybowski $^{2}$ and Louis-S Bouchard ${ }^{1,3 *}$ \\ ${ }^{1}$ Dept. of Chemistry and Biochemistry, University of California, Los Angeles, CA 90095-1569 USA \\ ${ }^{2}$ Dept. of Chemistry and Biochemistry, University of Delaware, Newark, DE 19761-2522 USA \\ ${ }^{3}$ California NanoSystems Institute, UCLA, Los Angeles, CA 90095-1569 USA \\ *Corresponding author: Louis-S Bouchard (email: bouchard@chem.ucla.edu)
}

\begin{abstract}
In this study we present an alternative approach to separating contributions to the NMR shift originating from the Knight shift and chemical shielding by a combination of experimental solid-state NMR results and $a b$ initio calculations. The chemical and Knight shifts are normally distinguished through detailed studies of the resonance frequency as function of temperature and carrier concentration, followed by extrapolation of the shift to zero carrier concentration. This approach is time-consuming and requires studies of multiple samples. Here, we analyzed ${ }^{207} \mathrm{~Pb}$ and ${ }^{125}$ Te NMR spin-lattice relaxation rates and NMR shifts for bulk and nanoscale PbTe. The shifts are compared with calculations of the ${ }^{207} \mathrm{~Pb}$ and ${ }^{125} \mathrm{Te}$ chemical shift resonances to determine the chemical shift at zero charge carrier concentration. The results are in good agreement with literature values from carrier concentration-dependent studies. The measurements are also compared to literature reports of the ${ }^{207} \mathrm{~Pb}$ and ${ }^{125} \mathrm{Te}$ Knight shifts of $n$ and $p$-type $\mathrm{PbTe}$ semiconductors. The literature data have been converted to the currently accepted shift scale. We also provide possible evidence for the "self-cleaning effect" property of PbTe nanocrystals whereby defects are removed from the core of the particles, while preserving the crystal structure.
\end{abstract}


Key Words ${ }^{207} \mathrm{~Pb}$ NMR; ${ }^{125} \mathrm{Te} \mathrm{NMR}$; lead telluride; nanocrystals; self-cleaning effect; Knight shift; chemical shift; spin-lattice relaxation.

\section{Introduction}

Lead telluride is a narrow gap semiconductor long used for thermoelectric applications. Snyder and co-workers ${ }^{1}$ recently provided an overview of the scientific and technological history of this material. The overview begins "during the Cold War and Space Race of the middle part of the $20^{\text {th }}$ century" and concludes by discussing how band structure engineering to improve overall thermoelectric efficiency has recently been driving this field of research. Among the many ways to interrogate the state of semiconductors such as $\mathrm{PbTe}$, nuclear magnetic resonance (NMR) of both nuclei has been extremely valuable in assessing the electronic state ${ }^{2-7}$. For example, the Knight shift ${ }^{8}$, which probes the interaction of nuclear spins with conduction band carriers (electrons or holes), provided a direct measure of carrier concentration. This readout can be performed even on samples that are not amenable to transport studies. Such readouts could, in principle, aid in the development of novel materials which cannot be produced as high quality thin films or single crystals. This potentially important technological application is currently hampered, however, by our inability to separate Knight shift from chemical shift.

The Knight shift is typically measured relative to the resonance of the nucleus of interest in a "carrier-free sample", thus the importance of the reference point. Nuclei such as ${ }^{207} \mathrm{~Pb}$ experience strong chemical shielding contributions to the overall shift. These early studies report the Knight shifts as the magnetic field for the resonance of ${ }^{207} \mathrm{~Pb}$ and ${ }^{125} \mathrm{Te}$ nuclei at a given radio frequency (RF). In these terms, the Knight shift, $K$, is given by ${ }^{6}$

$$
K=\left(H_{r}-H_{s}\right) / H_{s}
$$


where $H_{r}$ is the magnetic field at which resonance of a sample free of charge carriers occurs, i.e. a sample whose resonance is governed only by the chemical shielding ${ }^{9,10} . H_{s}$ is the resonance field of a "real sample [...] at constant frequency". The chemical-shielding reference field, $H_{r}$, is usually determined indirectly by extrapolating the dependence of $H_{s}$ on carrier concentration (measured by some other technique) back to zero carrier concentration.

There are some challenges in determining the chemical shielding and expressing magnetic field values reported in the earlier literature in terms of the current convention for chemical shifts ${ }^{11}$

$$
\delta(p p m)=\left\{\frac{\left(\text { sample }^{-} \text {reference }\right)}{\text { reference }}\right\} \times 10^{6}
$$

One challenge is the accuracy with which the resonance field can be read from graphs in the older literature. Another issue arises from the temperature dependence of the chemical shifts and Knight shifts. The chemical shifts of diamagnetic lead compounds often have a strong dependence on temperature. As an example, the ${ }^{207} \mathrm{~Pb}$ resonance of lead nitrate is routinely used as a NMR thermometer because of the $0.76 \mathrm{ppm} / \mathrm{K}$ change of the resonance position with temperature $^{12-19}$. Separating the temperature dependence of the Knight shift from that of the chemical shift can be done only for temperature studies with different samples of $n$ - and $p$-type semiconductors. This procedure is time-consuming and strongly depends on the quality of the samples and their characterization by transport measurements. The reliability of transport measurements in turn depends on sample quality.

Previous experiments focused on ${ }^{207} \mathrm{~Pb}$ and ${ }^{125} \mathrm{Te}$ NMR line positions as functions of carrier concentration and temperature; band structure calculations provided a qualitative understanding of the results and energy gap. The ${ }^{207} \mathrm{~Pb}$ and ${ }^{125} \mathrm{Te}$ chemical shifts relative to the resonance of a standard material are dependent on the local structure and electronic environment. 
As a result, chemical shift tensors provide qualitative trends that yield insights into the chemical bonding. The structure of $\mathrm{PbTe}$ is particularly simple, which allows one to compare experimentally-measured chemical shifts with first-principles calculations.

The nuclear magnetic relaxation of ${ }^{207} \mathrm{~Pb}$ and ${ }^{125} \mathrm{Te}$ reflects the dynamics of fluctuating magnetic fields in the vicinity of the nucleus. In semiconductors, relaxation is frequently dominated by spin-flip scattering with the conduction charge carriers. As such, the relaxation behavior is correlated with the Knight shift. The relaxation behavior of both ${ }^{207} \mathrm{~Pb}$ and ${ }^{125} \mathrm{Te}$ as a function of temperature confirms, as with the shifts, that the sample contains a distribution of electronic environments ${ }^{20}$.

\section{Experimental and Theoretical Methods}

The NMR data were acquired with a Bruker DSX-300 spectrometer operating at a frequency of $62.79 \mathrm{MHz}$ for ${ }^{207} \mathrm{~Pb}$ and $94.69 \mathrm{MHz}$ for ${ }^{125} \mathrm{Te}$. A standard Bruker X-nucleus wideline probe with a 5-mm solenoid coil was used with a static polycrystalline sample of $\mathrm{PbTe}$. The PbTe samples were obtained from Sigma Aldrich (avg. size: $84 \%$ smaller than $177 \mu \mathrm{m}$ and 13\% between 177 and $354 \mu \mathrm{m}$ ) and Alfa Aesar. The PbTe particle size was small enough to avoid RF skin-depth effects at these frequencies. The sample was confined to the length of the RF coil. The ${ }^{207} \mathrm{~Pb} \pi / 2$ pulse width was $4.5 \mu$ s, and the ${ }^{125} \mathrm{Te} \pi / 2$ pulse width was $4 \mu$ s. We have verified that these RF pulses excited the entire NMR resonances. Magic-angle-spinning $(\mathrm{MAS})^{21-23}$ spectra were acquired on the same sample with a standard Bruker MAS probe using a 4-mm outside diameter zirconia rotor with a sample spinning rate of $10 \mathrm{kHz}$. The ${ }^{207} \mathrm{~Pb}$ and ${ }^{125} \mathrm{Te} \pi / 2$ pulse widths for the MAS experiments were $4.4 \mu \mathrm{s}$. To minimize pulse ringdown effects, spectral data were acquired using a spin-echo sequence $\left.[\pi / 2)_{\mathrm{x}}-\tau-\pi\right)_{\mathrm{y}}-\tau-$ acquire $]$ with 
the echo delay, $\tau$, set to $20 \mu \mathrm{s}$. Data for determining the spin-lattice relaxation times $\left(T_{I}\right)$ were acquired with a saturation-recovery technique ${ }^{24}$. The ${ }^{207} \mathrm{~Pb}$ and ${ }^{125} \mathrm{Te}$ chemical shift scales were calibrated using the unified $\Xi$ scale ${ }^{11}$, relating the nuclear resonance frequency to the ${ }^{1} \mathrm{H}$ resonance of dilute tetramethylsilane in $\mathrm{CDCl}_{3}$ at a frequency of $300.13 \mathrm{MHz}$. The reference for ${ }^{207} \mathrm{~Pb}$ is tetramethyllead (TML) and the reference for ${ }^{125} \mathrm{Te}$ is dimethyltelluride. The two chemical shift scales were experimentally verified by acquiring spectra of lead nitrate ${ }^{16-18}$ and telluric acid ${ }^{25}$. We also note the existence of alternative ${ }^{125} \mathrm{Te}$ absolute chemical shielding scales and comparisons to density functional theory (DFT) predictions ${ }^{26-28}$. A relaxation delay of at least five times $T_{l}$ was used in each experiment to allow full recovery of the magnetization.

Quantum calculations were performed with the Amsterdam Density Functional theory (ADF) suite of software ${ }^{29-31}$ on models of the local PbTe environment. Calculations employed the Becke88-Perdew86 generalized gradient approximation (BP86) functional ${ }^{32-33}$ and a triplezeta double polarized (TZ2P) basis set. The calculations included relativistic effects by the zero order regular approximation (ZORA) to the two-component Dirac equation with spin-orbit coupling $^{34-35}$. NMR chemical shieldings were calculated with the NMR module ${ }^{36}$ associated with the ADF program package. The ${ }^{207} \mathrm{~Pb}$ chemical shifts are reported relative to tetramethyllead (TML), whose isotropic chemical shift was calculated at the same level of theory. The ${ }^{125}$ Te chemical shifts are reported relative to dimethyltelluride.

\section{Results and Discussion}

\section{Chemical Shifts}

With modern NMR spectrometers, the ${ }^{207} \mathrm{~Pb}$ resonance frequency is typically measured in a magnetic field of a fixed strength. The measured NMR shift includes contributions from 
chemical shielding and the Knight shift. For diamagnetic insulators, the chemical shift of ${ }^{207} \mathrm{~Pb}$ can range up to $12,000 \mathrm{ppm}$ relative to tetramethyllead ${ }^{37}$. Similarly, the range of chemical shifts of ${ }^{125} \mathrm{Te}$ is approximately $5,000 \mathrm{ppm}$. The ${ }^{207} \mathrm{~Pb}$ Knight shifts are among the largest known shifts for nontransition materials, on the order of $1 \%$ for a carrier concentration of $10^{19} \mathrm{~cm}^{-3}$ in $n$ - and p-type $\mathrm{PbTe}$ semiconductors ${ }^{5}$ (although the ${ }^{125} \mathrm{Te}$ shift remains small in these materials). As mentioned previously, the contributions of the two components to the overall shift can be separated because the Knight shift is experimentally determined by measuring the resonance position relative to that of a "carrier-free sample".

Lead telluride has been investigated with temperature-dependent NMR spectroscopy by several groups ${ }^{2-7}$. The theoretical challenge has been to explain the large Knight shift of ${ }^{207} \mathrm{~Pb}$ arising from both the $s$-like holes and $p$-like conduction electrons. As discussed by Sapoval and co-workers ${ }^{4,5}$, the early theory suggested that only $s$-like electrons give rise to a large Knight shift. They noted that more comprehensive models with the full expression for hyperfine coupling indicated that large Knight shifts can also arise from the spin-orbit term for $p$-type electrons and that a dipolar field from the electron, "even in cubic crystals", can arise due to the spin-orbit term.

These early studies typically reported Knight shifts as the magnetic field for the resonance of ${ }^{207} \mathrm{~Pb}$ nuclei at a given radiofrequency according to Eq. (1). These experiments were done in the swept-field/constant frequency mode. The resonance fields of ${ }^{207} \mathrm{~Pb}$ at a frequency of $7.5056 \mathrm{MHz}$ as a function of the carrier concentration for a series of $n$ - and $p$-type $\mathrm{PbTe}$ at $1.3 \mathrm{~K}$ have been measured and extrapolated back to a field of 8436 gauss for a hypothetical sample of zero carriers ${ }^{2,5}$ to yield the chemical shift of $\mathrm{PbTe}$ at that temperature. A different study ${ }^{3}$ reported the resonance fields of ${ }^{207} \mathrm{~Pb}$ at a frequency of $10.0 \mathrm{MHz}$ as a function 
of the carrier concentration for a series of $n$ - and $p$-type PbTe samples from $77 \mathrm{~K}$ to $465 \mathrm{~K}$. This work indicated a field of 11,216 gauss as the zero-charge-carrier resonance field at $300 \mathrm{~K}$. The combination of these two studies provides the temperature dependence of the chemical shift of PbTe.

As the resonance frequency is directly proportional to the magnetic field ${ }^{8}$, the observed resonance frequency can be scaled to a different magnetic field. This process was used in many early studies for comparison of experimental results. To express these experimental results from earlier studies in terms of current conventions ${ }^{11}$ and to compare them directly with the experimental results reported in the present work, we have converted these earlier results to the appropriate frequencies that would be observed in a magnetic field of $7.04925 \mathrm{~T}$ in which the protons of tetramethylsilane are observed at a frequency of $300.130 \mathrm{MHz}$. These frequencies at 7.04925 $\mathrm{T}$ were then converted to parts per million $(\mathrm{ppm})$ relative to the reference material by use of Eq. (2) and the unified $\Xi$ scale as described in Ref. (11). (Examples of this conversion process are explicitly shown in the Supporting Information section.) Sapoval's extrapolation ${ }^{4}$ for the ${ }^{207} \mathrm{~Pb}$ chemical shift of $\mathrm{PbTe}$ at $1.3 \mathrm{~K}$ can be expressed as $-420.8 \mathrm{ppm}$. The variabletemperature study ${ }^{3}$ yields a ${ }^{207} \mathrm{~Pb}$ chemical shift of $970.8 \mathrm{ppm}$ at $300 \mathrm{~K}$.

The ${ }^{207} \mathrm{~Pb}$ spectrum of a static polycrystalline sample of PbTe at $295 \mathrm{~K}$ is shown in Fig. 1(a). The resonance line shape is clearly asymmetric. Such an asymmetrical feature likely arises from interaction with conduction carriers ${ }^{38-47}$. In a prior study ${ }^{48,49}$, asymmetric resonance lines were attributed to an inhomogeneous distribution of defects yielding different charge carrier concentrations in different microcrystalline regions, giving a range of Knight shifts for spins in these different environments. Specifically, the ${ }^{207} \mathrm{~Pb}$ Knight shift in the case of PbTe with low $p$ type carrier concentrations has been reported ${ }^{5}$ to go as $n^{1 / 3}$, where $n$ is the charge carrier 
concentration. The tensorial nature of the electron-nuclear hyperfine interaction, namely the contributions from dipolar and orbital terms in a material with $p$-band charge carriers, could also contribute to the asymmetry of the line shape ${ }^{50}$.

In the ${ }^{207} \mathrm{~Pb}$ spectrum of the static sample shown in Fig. 1(a), the experimental resonance extends from roughly $1,600 \mathrm{ppm}$ to about $-500 \mathrm{ppm}$, with a maximum intensity at ca. $875 \mathrm{ppm}$. In Fig. 1(a), the spectrum of the sample obtained under magic-angle spinning (MAS) has essentially the same structure, but it is slightly shifted to higher frequencies, with the maximum intensity at $\sim 1,000 \mathrm{ppm}$. If the least shielded areas of the sample correspond to lower carrier concentrations, the results of Fig. 1(a) suggest a chemical shift at $295 \mathrm{~K}$ of between 1,200 and 1,400 ppm. For comparison with the earlier literature (as converted for the present work), Sapoval and co-workers ${ }^{2,5}$ report a chemical shift of $-420.8 \mathrm{ppm}$ at a temperature of $1.3 \mathrm{~K}$. The later work by Senturia, et al. ${ }^{3}$ gives a chemical shift of $970.8 \mathrm{ppm}$ at $300 \mathrm{~K}$. From these observations, we conclude that the ${ }^{207} \mathrm{~Pb}$ chemical shift of $\mathrm{PbTe}$ is in the range of 1,000 to 1,400 ppm, and may depend on how the experiment is carried out, including the analysis of the variation with charge carrier concentration ${ }^{38,39,49}$.

These "experimental" ${ }^{207} \mathrm{~Pb}$ chemical shifts for $\mathrm{PbTe}$, obtained from extrapolation to zero concentration, for the carrier density can be compared with the chemical shift calculated with DFT including relativistic effects through the zero order regular approximation (ZORA) that includes scalar and spin-orbit effects. For the lead chemical shifts, the model is a lead atom surrounded by an octahedral array of six tellurium atoms; six hydrogen atoms were included to compensate the major charge on the cluster. For chemical shift referencing, the lead chemical shielding of tetramethyllead (TML) was calculated at the same level of theory. The results in Table I indicate that the diamagnetic contribution to the chemical shielding of lead is virtually 
independent of the $\mathrm{Pb}-\mathrm{Te}$ distance, reflecting the major contributions from lead core electrons. This relative constancy of the diamagnetic contribution has also been observed in calculations of the ${ }^{207} \mathrm{~Pb}$ chemical shifts of the lead dihalides and the lead tetrahalides ${ }^{51}$. The paramagnetic and spin-orbit contributions, on the other hand, depend on the $\mathrm{Pb}$-Te bond distance, so that the total chemical shift is dependent on the $\mathrm{Pb}-\mathrm{Te}$ bond distance, as can be seen by the results in the last column of Table I. The calculated shift of $1,550 \mathrm{ppm}$ for a PbTe bond length of $323 \mathrm{pm}$, matching the bond length reported in the crystal structure ${ }^{52,53}$, is in agreement with the shifts observed in the commercial PbTe sample containing native defects and impurities.

For the tellurium resonance, the line shape of the commercial sample is relatively symmetric, as can be seen in Fig. 1(b), with a mean resonance position of $-1,176 \mathrm{ppm}$. The resonance covers a range from roughly $-1,150 \mathrm{ppm}$ to $-1,225 \mathrm{ppm}$. In this case it is difficult to know the chemical shift by assuming it is at the "edge" of the pattern, but a rough estimate would be around $-1,150 \mathrm{ppm}$. The earlier literature, in a study ${ }^{3}$ of $n$ - and $p$-type $\mathrm{PbTe}$ as a function of the carrier concentration, indicated a magnetic field of 9,689 gauss as the resonance field at $13.0 \mathrm{MHz}$ for the ${ }^{125} \mathrm{Te}$ chemical shift at $77 \mathrm{~K}$. This corresponds to a chemical shift of $1,147 \mathrm{ppm}$ at $77 \mathrm{~K}$. The measured ${ }^{125} \mathrm{Te}$ shift of $-1,176 \mathrm{ppm}$ suggests the sample is a $p$-type semiconductor, in agreement with the ${ }^{207} \mathrm{~Pb}$ result. According to Balz et al. ${ }^{54}$, the shape of ${ }^{125} \mathrm{Te}$ MAS spectra (e.g. Fig. 1(b)) could be explained using $J\left({ }^{207} \mathrm{~Pb},{ }^{125} \mathrm{Te}\right)=2150 \mathrm{~Hz}$ for various isotopomers of a $\mathrm{TePb}_{6}$ species to account for the width and shoulders in the spectrum. The chemical shift of the ${ }^{125} \mathrm{Te}$ resonance has been explained in Ref. (56).

A model to calculate the ${ }^{125} \mathrm{Te}$ chemical shift is a cluster consisting of a tellurium atom surrounded by an octahedral array of six lead atoms. To terminate the structure, fluorine atoms are appended to the lead atoms. The chemical shift as a function of $\mathrm{Pb}-\mathrm{Te}$ distance is given in 
Table II, reported as the shift relative to that of neat dimethyltelluride ${ }^{11}$ calculated at the same level of theory. The predicted chemical shift changes with the internuclear distance, but not to the extent that the lead chemical shift changes with internuclear distance. Over the same range of bond distance, the lead shift changes roughly five times as rapidly as the tellurium chemical shift. This theoretical observation is consistent with the generally narrower ${ }^{125} \mathrm{Te}$ band as compared to the changes of lead chemical shifts. The predicted ${ }^{125} \mathrm{Te}$ chemical shift is $-1,134$ ppm for the measured bond length. This is in good agreement with the experimentally observed value of $-1,176 \mathrm{ppm}$.

\section{Spin-lattice Relaxation}

The spin-lattice relaxation time, $T_{1}$, is a useful probe of electronic structure in semiconductors. The ${ }^{207} \mathrm{~Pb}$ and ${ }^{125} \mathrm{Te}$ spin-lattice relaxation behaviors in saturation-recovery experiments have been measured as a function of temperature. A typical recovery of ${ }^{207} \mathrm{~Pb}$ nuclear longitudinal magnetization at $295 \mathrm{~K}$ is shown in Fig. 2, together with a stretched exponential function representing the "best fit" to the data. Each data point in the graph of Fig. 2 represents the integral of the NMR spectrum across the entire range of frequencies. Therefore, relaxation in regions with different Knight shifts are included, yielding a distribution of relaxation times. The data are not fit by a single exponential as would be expected if all nuclei in the sample recovered with the same rate constant. Figure 3 compares the fully relaxed ${ }^{207} \mathrm{~Pb}$ spectrum with a partially relaxed spectrum $(\tau=10 \mathrm{~ms})$ of the sample. It is clear from this figure that the relaxation is inhomogeneous, with the regions of the line shape at more negative shifts being relaxed faster than those at less negative shifts. Treating the most intense spectral feature

around $875 \mathrm{ppm}$ as a single isochromat yields a ${ }^{207} \mathrm{~Pb} T_{l}$ of approximately $27 \mathrm{~ms}$, while the more 
rapidly recovering part in the range $700 \mathrm{ppm}$ to $-500 \mathrm{ppm}$ has a $T_{1}$ around $12.7 \mathrm{~ms}$. As the rate of relaxation, $1 / T_{1}$, is known to be proportional to the carrier density ${ }^{20,38,48}$, the variation of $T_{1}$ across the line shape suggests that the line shape arises from an inhomogeneous distribution of defects, creating domains with differing carrier concentrations ${ }^{46,47}$. The shorter $T_{1}$ and the increased Knight shift to lower frequency are consistent with an increased carrier concentration $^{20,46}$. This shift to lower frequency of this sample appears to place it in the range of resonances for $p$-type $\mathrm{PbTe}$ semiconductors ${ }^{3}$, as mentioned above.

As a result of the distribution of defects, the ${ }^{207} \mathrm{~Pb}$ spin-lattice relaxation data is best characterized by a Kohlrausch (stretched-exponential) function

$$
M(t)=M_{0}\left(1-\exp \left(-\frac{t}{T_{1}}\right)^{\beta}\right)
$$

The application of this empirical model to physical systems, including NMR relaxation, is well established $^{56}$. It is a natural choice for $\mathrm{PbTe}$, which is characterized by an inhomogeneous distribution of carrier concentrations and defects. The results of this analysis of the ${ }^{207} \mathrm{~Pb}$ relaxation rate are shown in Fig. 4. The fitting of this plot gives an activation energy of 166 $\mathrm{meV}(16.02 \mathrm{~kJ} / \mathrm{mol})$. This value is virtually identical to the $160 \mathrm{meV}(15.4 \mathrm{~kJ} / \mathrm{mole})$ activation energy for processes that induce relaxation of ${ }^{205} \mathrm{Tl}$ in semiconducting $\mathrm{Tl}_{2} \mathrm{Se}^{57}$.

The ${ }^{125} \mathrm{Te}$ spin-lattice relaxation $\left(T_{I}\right)$ data from a saturation-recovery experiment at $295 \mathrm{~K}$ are shown in Fig. 2, as presented next to ${ }^{207} \mathrm{~Pb}$ results. As is the case for ${ }^{207} \mathrm{~Pb}$, the relaxation clearly cannot be described as an exponential approach to equilibrium. The result of fitting these data to a stretched exponential function is shown in Fig. 5. (For the interested reader, a biexponential analysis of the same data is provided in the Supporting Information section.) In contrast to the results obtained by fitting a stretched-exponential to the ${ }^{207} \mathrm{~Pb}$ relaxation data, the ${ }^{125}$ Te results do not follow Arrhenius behavior with temperature. The low-temperature behavior 
indicates that at temperatures below about $250 \mathrm{~K}$, another relaxation mechanism becomes sufficiently effective that it dominates relaxation. The limited data in this region indicate an activation energy of about $62.72 \mathrm{meV}$ for this process. At higher temperatures a second relaxation mechanism becomes dominant, characterized by an activation energy of $159.24 \mathrm{meV}$. This second mechanism has, within experimental error, the same activation energy as was observed through the ${ }^{207} \mathrm{~Pb}$ spin-lattice relaxation. It is clear that relaxation of ${ }^{125} \mathrm{Te}$ in this temperature region and ${ }^{207} \mathrm{~Pb}$ are effected by the same processes, an interaction with the conduction charge carriers, but that ${ }^{125} \mathrm{Te}$ experiences another mechanism at low temperatures that is not seen to affect the ${ }^{207} \mathrm{~Pb}$ relaxation.

\section{Temperature-Dependent Changes in Line Shapes}

As discussed in the Introduction, there are challenges in accurately determining the "experimental" chemical shift from these earlier studies of PbTe. The challenges include the extrapolation to or estimation of the resonant magnetic field for a sample of zero charge carrier concentration, reading such values from graphs in the literature, and dealing with the effects of temperature upon the shifts. There are also challenges in accurately calculating the chemical shifts of such heavy nuclei that result in significant uncertainty in the predicted chemical shifts. As a result, the assignment of the $\mathrm{PbTe}$ used in this study as a $p$-type semiconductor based on comparisons with the ${ }^{207} \mathrm{~Pb}$ and ${ }^{125} \mathrm{Te}$ "experimental" chemical shifts obtained from the shifts of samples as a function of charge carrier concentration, while strongly suggestive, is still somewhat tenuous. The calculated chemical shifts, also strongly suggestive, do not clearly resolve the issue. The ${ }^{207} \mathrm{~Pb}$ shift of this sample as a function of temperature, shown in Fig. 6, exhibits the same behavior as that of the p-type samples given in Fig. 3 of Ref. (3). Increasing 
the temperature of $p$-type samples of $\mathrm{PbTe}$ moves the resonant magnetic field to lower values (corresponding to increases in frequencies at a fixed magnetic field). The opposite behavior is observed for $n$-type samples. This temperature dependence of the ${ }^{207} \mathrm{~Pb}$ shift confirms the sample used in this study is indeed a $p$-type semiconductor.

Although the ${ }^{125} \mathrm{Te}$ chemical shifts at $77 \mathrm{~K}$ have been reported as a function of carrier concentration $^{3}$, these early studies of PbTe did not provide any results for the ${ }^{125} \mathrm{Te}$ chemical shift as a function of temperature for comparison with those obtained in this study. The dependence of the ${ }^{125} \mathrm{Te}$ shift on temperature for the $p$-type sample of PbTe used in this work is shown in Fig. 7. Periodic checks of the sample integrity were made during the variable temperature (VT) studies. This was done by acquiring a one-dimensional ${ }^{207} \mathrm{~Pb}$ spectrum of the sample at $295 \mathrm{~K}$ after various stages of the VT study. These results are shown in Fig. 8. A change in the spectrum appeared after elevating the temperature to $423 \mathrm{~K}$ with the ${ }^{207} \mathrm{~Pb}$ spectral features moving to a slightly higher NMR frequency. This change is likely due to some annealing of the native defects. The effect is to move the resonance position closer to that of the true chemical shift, as shown in Fig. 8. The annealing temperature used in our study is lower than those used in the annealing study of Hewes et al. ${ }^{58}$. Due to the high sensitivity of the NMR experiment to sample defects, homogeneity and carrier concentration (see also, Ref. 57), accuracy in such estimates may require the use of one or more annealing cycles. The lack of sample annealing may lead to systematic errors in the estimation of the chemical shift comparable to that shown in Fig. 10. In addition to the observed ${ }^{207} \mathrm{~Pb}$ shift, the changes upon annealing will also manifest themselves in the ${ }^{207} \mathrm{~Pb}$ spin-lattice relaxation time.

Another source of systematic errors arises from the powdering of the samples and can alter the NMR shift (and consequently, the spin-lattice relaxation rate). The early NMR studies ${ }^{2-}$ 
${ }^{7}$ of semiconductors usually determined the carrier concentrations on single crystal specimens and then powdered the samples for the NMR measurements. Hewes et al. ${ }^{6}$ noted that the powdering process can alter the carrier concentration. For lead telluride, differences in carrier concentration were noted between those samples cleaved with a razor blade and those crushed with a mortar and pestle ${ }^{6,58}$. The annealing study ${ }^{58}$ of $n$ - and $p$-type PbTe should generally help in determining error propagation arising from changes in carrier concentration, as carrier concentration as function of annealing temperature in the range of $452 \mathrm{~K}$ and $1,000 \mathrm{~K}$ are reported. In previous studies on PbTe samples ball milled for $60 \mathrm{~min}^{59,60}$, the crystallinity was preserved, but carrier concentration decreased. The decrease in carrier concentration in nanocrystalline samples is referred as the "self-cleaning" process of the bulk ${ }^{61-64}$ whereby defects are pushed toward the surface. The nanoparticles possess fewer defects than the corresponding bulk material. Because of self-cleaning in nanoparticles, ball-milling would be expected to decrease spin lattice relaxation rate $\left(1 / T_{1}\right)$, as shown in Fig. 5 for the finest ball milled $\mathrm{PbTe}(83$ $\mathrm{nm}$ ). The self-cleaning process is directly related to the observed increase in the activation energy in the high and low temperature regime $(<250 \mathrm{~K})$. In Fig. 9, powder x-ray diffraction (PXRD) spectra for powdered PbTe ingots (Alfa Aesar, Ward Hill, MA) mortar and pestle versus ball milled (83 nm, $60 \mathrm{~min}$. ball milling) samples confirm that crystallinity is indeed preserved. However, the carrier concentration in these samples appears to change, as evidenced by the increased $T_{1}$ time with decreasing particle size (Table III).

Atomic self-diffusion of $\mathrm{Pb}$ and $\mathrm{Te}$ has been investigated ${ }^{65}$ in lead telluride at temperatures in the range $793-1,000 \mathrm{~K}$ with use of radioactive tracers. The self-diffusion coefficients reported were quite high: $2.9 \times 10^{-5} \mathrm{~cm}^{2} / \mathrm{sec}$ for $\mathrm{Pb}$ and $2.7 \times 10^{-6} \mathrm{~cm}^{2} / \mathrm{s}$ for Te. While the activation energy for diffusion of $\mathrm{Pb}$ was dependent upon the stoichiometry of the 
sample, the lowest measured activation for both $\mathrm{Pb}$ and $\mathrm{Te}$ at $100 \mathrm{~kJ} / \mathrm{mole}$ is still much higher than the activation energies found in the current investigation. Thus, the relaxation mechanism in the high-temperature regime cannot be due to atomic diffusion. The likely mechanism involves interactions of nuclear moments with thermally activated charge carriers ${ }^{46,47,57}$. The low-temperature process that affects the ${ }^{125} \mathrm{Te}$ relaxation can be, like for $\mathrm{TlTaS}_{3}$, due to spin diffusion to localized electron spins.

Magic-angle spinning (MAS) does not lead to a significant narrowing of the ${ }^{207} \mathrm{~Pb}$ resonance, as shown in Fig. 1(a). The shift to higher frequency is consistent with an increased temperature in the MAS rotor resulting from the sample spinning at $10 \mathrm{kHz}^{38,41,66}$. A measurement of the ${ }^{207} \mathrm{~Pb}$ NMR shift of lead nitrate ${ }^{12-19}$ at an ambient temperature of $295 \mathrm{~K}$ indicates a sample temperature of $306 \mathrm{~K}$ at this rotation rate. However, the magnitude of the change in the shift at $140 \mathrm{ppm}$ for the MAS spectrum is much larger than that expected from frictional heating of the MAS rotor alone. Such an effect of similarly large shifts in MAS spectra of conductive samples has been explained ${ }^{66}$ for spectra of $\mathrm{CuI}$. This shift to higher frequency for the ${ }^{207} \mathrm{~Pb}$ resonance of a static sample of lead telluride as the temperature is increased is demonstrated in Fig. 6. MAS increases the magnitude of this shift in conductive samples.

MAS also does not substantially narrow the ${ }^{125}$ Te resonance, as shown in Fig. 1(b). The rotation rate of $10 \mathrm{kHz}$ is larger than the ${ }^{125} \mathrm{Te}$ static line width of $4.7 \mathrm{kHz}$. As MAS does not result in a manifold of spinning sidebands in either the ${ }^{207} \mathrm{~Pb}$ or ${ }^{125} \mathrm{Te}$ MAS spectra, the broadening of the spectral resonance is not due solely to an orientational dependence of chemical shifts (or Knight shifts). While Figure 7 shows that the ${ }^{125} \mathrm{Te}$ resonance of the static sample exhibits the same shift to increasing frequency as the temperature is raised as observed with the ${ }^{207} \mathrm{~Pb}$ spectra shown in Figure 6, the ${ }^{125} \mathrm{Te}$ MAS spectrum in Figure 1(b) shows a slight 
narrowing of the resonance with a shift of the first moment of the resonance to lower frequency. This likely arises due to the averaging of anisotropic interactions.

\section{Conclusions}

Unless samples of zero Knight shift (i.e. with low carrier concentration) are available as reference, the chemical and Knight shifts are normally distinguished through detailed studies of the resonance frequency as function of temperature and carrier concentration, followed by extrapolation of the shift to zero carrier concentration. In order to overcome this timeconsuming approach we presented here a combined NMR and DFT method to separate these two contributions. A second advantage of the approach is it enables studies of specific samples with fixed physical properties whose carrier concentrations cannot easily be altered. Calculations of the chemical shift of PbTe based on model structures are in agreement with experiment. Experiments confirm that $\mathrm{PbTe}$ consists of a distribution of crystallites having differing densities of charge carriers, resulting in an asymmetric line shape due to a distribution of Knight shifts in various domains of the sample. Our results suggest that the sample studied was $p$-type, in agreement with previous carrier concentration-dependent studies of $n$ - and $p$-type PbTe semiconductor.

Relaxation studies show that the sample exhibits dynamics that arise from a range of charge carrier densities. In the high-temperature regime, relaxation is governed by interaction with thermally activated charge carriers. For ${ }^{125} \mathrm{Te}$ at temperatures below about $250 \mathrm{~K}$, a second mechanism dominates spin-lattice relaxation, which we postulate may be due to spin diffusion to isolated electron spins. The low temperature regime is also suggestive that a second mechanism 
affects ${ }^{207} \mathrm{~Pb}$ relaxation. Thus, interaction with both mobile carriers and fixed centers contributes to relaxation in $\mathrm{PbTe}$.

The literature data on $\mathrm{PbTe}$ have been converted to the currently accepted shift scale. We also provide possible evidence for the "self-cleaning effect" property of $\mathrm{PbTe}$ nanocrystals whereby defects are removed from the core of the particles, while preserving the crystal structure.

\section{Acknowledgments}

This material is based upon work supported by the National Science Foundation through Grant CHEM0956006 to C.D. The work at UCLA (L.-S. B.) was supported by Defense Advanced Research Project Agency (DARPA), award no: N66001-12-1-4034. L.-S. B. acknowledges useful discussions with Mercouri G. Kanatzidis.

\section{References}

(1) LaLonde, A. D.; Pei, Y.; Wang, H.; Snyder, G. J. Lead telluride alloy thermoelectrics. Materials Today 2011, 14, 526 - 532 .

(2) Sapoval, B. Knight Shifts and Band Structure in Lead Telluride by Helicon-Nuclear-Spin Interaction. J. Phys. 1968, (Paris) Suppl. 29, C4-133 - C4-136.

(3) Senturia, S. D.; Smith, A. C; Hewes, C. R.; Hofmann, J. A.; Sagalyn, P. S. Knight Shfits and Band Structure in the Lead-Salt Semiconductors. Phys. Rev. B 1970 , 1, 4045 - 4057.

(4) Sapoval, B.; Leloup, J. Y. Knight Shift in Multivalley Semiconductors. I. Theory of Contact, Orbital, and Dipolar Shift and Relativistic Effects. Phys. Rev. B 1973, 7, 5272 - 5276.

(5) Leloup, J. Y.; Sapoval, B; Martinez, G. Knight Shift in Multivalley Semiconductors. II. Determination of the Hyperfine Coupling Constants in $N$ - and P-Type PbSe and PbTe. Phys. Rev. B 1973, 7, 5276 - 5284. 
(6) Hewes, C. R.; Adler, M. S.; Senturia, S. D. Nuclear-Magnetic-Resonance Studies in PbTe and $\mathrm{Pb}_{1-\mathrm{x}} \mathrm{Sn}_{\mathrm{x}} \mathrm{Te}$ : An Experimental Determination of $\vec{k} \cdot \vec{p}$ Band Parameters and Magnetic Hyperfine Constants. Phys. Rev. B 1973, 7, 5195 - 5211.

(7) Martinez, G.; Schlüter, M.; Cohen, M. L. Electronic structure of PbSe and PbTe. I. Band structures, densities of states, and effective masses. Phys. Rev. B 1975, 11, 651-659.

(8) Knight, W. D. Nuclear magnetic resonance shift in metals. Phys. Rev. 1949, 76,1259 -1260.

(9) Proctor, W. G.; Yu, F. C. The Dependence of a Nuclear Magnetic Resonance Frequency upon Chemical Compound. Phys. Rev. 1950, 77, 717-717.

(10) Dickenson, W. C. Dependence of the F19 Nuclear Resonance Position on Chemical Compound. Phys. Rev. 1950, 77, 736-737.

(11) Harris, R. K.; Becker, E. D.; Cabral de Menezes, S. M.; Goodfellow, R.; Granger, P. NMR Nomenclature. Nuclear Spin Properties and Conventions for Chemical Shifts. Pure Appl. Chem. 2001, 73, 1795-1818.

(12) Bielecki, A.; Burum, D. P. Temperature dependence of ${ }^{207} \mathrm{~Pb}$ MAS spectra of solid lead nitrate. An accurate, sensitive thermometer for variable-temperature MAS. J. Magn. Reson. A, 1995, 116, $215-220$.

(13) Ferguson, D. B.; Haw, J. F. Transient methods for in situ NMR of reactions on solid catalysts using temperature jumps. Anal. Chem. 1995, 67, $3342-3348$.

(14) van Gorkom, L. C. M.; Hook, J. M.; Logan, M. B.; Hann, J. V.; Wasylishen, R. E. Solidstate lead-207 NMR of lead(II) nitrate: Localized heating effects at high magic angle spinning speeds. Magn. Reson. Chem. 1995, 33, $791-795$.

(15) Mildner, T.; Ernst, H.; Freude, D.; ${ }^{207} \mathrm{~Pb}$ NMR detection of spinning-induced temperature gradients in MAS rotors. Solid State NMR 1995, 5, $269-271$.

(16) Neue, G.; Dybowski, C. Determining temperature in a magic-angle spinning probe using the temperature dependence of the isotropic chemical shift of lead nitrate. Solid State NMR 1997, 7, $333-336$.

(17) Takahashi, T.; Kawashima; H.; Sugisawa, H.; Baba, T. ${ }^{207} \mathrm{~Pb}$ chemical shift thermometer at high temperature for magic angle spinning experiments. Solid State NMR 1999, 15, 119 - 123.

(18) Beckmann, P. A.; Dybowski, C. A thermometer for nonspinning solid-state NMR spectroscopy. J. Magn. Res. 2000, 146, $379-380$.

(19) Dybowski, C.; Neue; G. Solid-state ${ }^{207} \mathrm{~Pb}$ NMR spectroscopy. Prog. NMR Spec. 2002, 41, $153-170$. 
(20) Levin, E. M.; Cook, B. A.; Ahn, K.; Kanatzidis, M. G.; Schmidt-Rohr, K. Electronic inhomogeneity and $\mathrm{Ag}: \mathrm{Sb}$ imbalance of $\mathrm{Ag}_{1-\mathrm{y}} \mathrm{Pb}_{18} \mathrm{Sb}_{1+\mathrm{z}} \mathrm{Te}_{20}$ high-performance thermoelectrics elucidated by ${ }^{125} \mathrm{Te}$ and ${ }^{207} \mathrm{~Pb}$ NMR. Phys. Rev. B 2009, 80, 115211.

(21) Andrew, E. R.; Bradbury, A.; Eades, R. G. Nuclear magnetic resonance spectra from a crystal rotated at high speed. Nature 1958, 182, 1659.

(22) Andrew, E. R.; Bradbury, A.; Eades, R. G. Removal of dipolar broadening of nuclear magnetic resonance spectra of solids by specimen rotation. Nature 1959, 183, 1802-1803.

(23) Lowe, I. J. Free induction decays of rotating solids. Phys. Rev. Lett. 1959, 2, 285-287.

(24) Farrar, T. C.; Becker, E. D. Pulse and Fourier Transform NMR, Introduction to Theory and Methods, Academic Press, New York, 1971.

(25) Collins, M. J.; Ripmeester, J. A.; Sawyer, J.F. CP/MAS ${ }^{125}$ Te NMR in Solids: An Example of ${ }^{125} \mathrm{Te}-{ }^{35,37} \mathrm{Cl} J$ Couplings. J. Am. Chem. Soc. 1987, 109, $4113-4115$.

(26) Jameson, C.J.; Jameson, A.K. Concurrent ${ }^{19} \mathrm{~F}$ and ${ }^{77} \mathrm{Se}$ or ${ }^{19} \mathrm{~F}$ and ${ }^{125} \mathrm{Te}$ NMR $T_{1}$ measurements for determination of ${ }^{77} \mathrm{Se}$ and ${ }^{125} \mathrm{Te}$ absolute shielding scales. Chem. Phys. Lett. 1987, 135, 254-259.

(27) Ruiz-Morales, Y.; Schreckenbach, G.; Ziegler, T. Calculation of ${ }^{125} \mathrm{Te}$ Chemical Shifts Using Gauge-Including Atomic Orbitals and Density Functional Theory. J. Phys. Chem. A 1997 101(2), 4121-4127.

(28) Fukuda, R.; Nakatsuji, H. Quasirelativistic theory for the magnetic shielding constant. III. Quasirelativistic second-order Møller-Plesset perturbation theory and its application to tellurium compounds. J. Chem. Phys. 2005, 123, 044101.

(29) Baerends, E.J.; Ziegler, T.; Autschbach, J.; Bashford, D.; Bérces, A.; Bickelhaupt, F.M.; Bo, C.; Boerrigter, P.M.; Cavallo, L.; Chong, D.P.; et al. ADF2012, SCM, Theoretical Chemistry, Vrije Universiteit, Amsterdam, The Netherlands, http://www.scm.com

(30) Jaworski, C.M.; Tobola, J.; Levin, E.M.; Schmidt-Rohr, K.; Heremans, J.P. Antimony as an amphoteric dopant in lead telluride. Phys. Rev. B 2009, 80, 125208.

(31) Nielsen, M.D.; Levin, E.M.; Jaworski, C.M.; Schmidt-Rohr, K. Chromium as resonant donor impurity in PbTe. Phys. Rev. B 2012, 85, 045210.

(32) Becke, A.D. Density-functional exchange-energy approximation with correct asymptotic behavior. Phys. Rev. A 1988, 38, 3098-3100.

(33) Perdew, J.P. Density-functional approximation for the correlation energy of the inhomogeneous electron gas. Phys. Rev. B 1986, 33, 8822-8824. 
(34) van Lenthe, E.; Baerends, E. J.; Snijders, J. G. Relativistic regular two-component Hamiltonians. J. Chem. Phys. 1993, 99, $4597-4610$.

(35) van Lenthe, E.; Ehlers, A.; Baerends, E. J. Geometry optimizations in the zero order regular approximation for relativistic effects. J. Chem. Phys. 1999, 110, $8943-8953$.

(36) Schreckenbach, G.; Ziegler, T.; Calculation of NMR Shielding Tensors Using GaugeIncluding Atomic Orbitals and Modern Density Functional Theory. J. Phys. Chem. 1995, 99, 606-611.

(37) Brevard C.; Granger, P. Handbook of High Resolution Multinuclear NMR, John Wiley and Sons, New York, 1981.

(38) Yesinowski, J. P.; Purdy, A. P.; Wu, H.; Spencer, M. G.; Hunting, J.; DiSalvo, F. J. Distributions of Conduction Electrons as Manifested in MAS NMR of Gallium Nitride. J. Am. Chem. Soc. 2006, 128 (15), 4952-4953.

(39) Holloway, H. Growth and application of epitaxial IV-VI compounds. J. Nonmetals 1973, 1, 347-354.

(40) Yesinowski, J.P. ${ }^{69,71} \mathrm{Ga}$ and ${ }^{14} \mathrm{~N}$ high-field NMR of gallium nitride films. Phys. Stat. Sol. 2005, 2 (7), 2399-2402.

(41) Yesinowski, J.P. Solid-state NMR of inorganic semiconductors. Top. Curr. Chem. 2012, 306, 229-312.

(42) Hirsch, M. J.; Holcomb, D. F. NMR Study of Si:As and Si:P near the metal-insulator transition. Phys. Rev. B 1986, 33, 2520-2529.

(43) Tripathi, G.S.; Das, L.K.; Misra, P.K.; Mahanti, S.D. Spin-orbit effects on Knight shift - a new contribution. Solid State Communications 1981, 38, 1207-1210.

(44) Misra, S.; Tripathi, G. S.; Misra, P. K. Theory of the Knight shift in narrow-gap semiconductor. J. Phys. C: Solid State Phys. 1987, 20, 277.

(45) Misra, C.M.; Tripathi, G.S. Knight Shift in PbTe AND Pb1-xSnxTe. Hyperfine Interactions 1990, 60, 619-622.

(46) Koumoulis, D.; Chasapis, T.C.; Taylor, R.E.; Lake, M.P.; King, D.; Jarenwattananon, N.N.; Fiete, G.A.; Kanatzidis, M.G.; Bouchard, L.S. NMR probe of metallic states in nanoscale topological insulators. Phys. Rev. Lett. 2013, 110, 026602.

(47) Taylor, R.E; Leung, B.; Lake, M.P.; Bouchard, L.S. Spin-lattice relaxation in bismuth chalcogenides. J. Phys. Chem. C. 2012, $116(32)$ 17300-17305. 
(48) Alexander, M. N.; Sagalyn, P. L.; Senturia, S. D.; Hewes, C. R. Nuclear spin relaxation study of the electronic structure of lead telluride. J. Nonmetals. 1973, 1, 251.

(49) Olekseeva, M. F.; Slinko, E. I.; Tovstyuk, K. D.; Handozhko, A. G.; Nuclear Magnetic Resonance in PbTe. Phys. Stat. Sol. 1974, $21(\mathrm{~A}), 759$ - 762.

(50) Yafet, Y.; Jaccarino, V. Nuclear Spin Relaxation in Transition Metals; Core Polarization. Phys. Rev. 1964, 133, A1630-A1637.

(51) Dmitrenko, O.; Bai, S.; Dybowski, C. Prediction of ${ }^{207} \mathrm{~Pb}$ NMR Parameters for the Solid Ionic Lead (II) Halides Using the Relativistic ZORA-DFT Formalism: Comparison with the Lead-containing Molecular Systems. Solid State Nucl. Magn. Reson. 2008, 34, 186 - 190.

(52) Noda, Y.; Ohba, S.; Sato, S.; Saito, Y. Charge distribution and atomic thermal vibration in lead chalcogenide crystals. Acta Crystallogr. B 1983, 39, 312 - 317.

(53) Noda, Y.; Masumoto, K.; Ohba, S.; Saito, Y.; Toriumi, Y. K.; Iwata, Y.; Shibuya, I.

Temperature dependence of atomic thermal parameters of lead chalcogenides, $\mathrm{PbS}, \mathrm{PbSe}$, and PbTe. Acta Crystallogr. C 1987, 43, 1443 - 1445.

(54) Balz, R.; Haller, M.; Hertler, W.E.; Lutz, O.; Nolle, A; Schafitel, R. ${ }^{125}$ Te NMR studies of indirect and direct dipole-dipole coupling in polycrystalline CdTe, HgTe, and PbTe. J. Magn. Reson. 1980, 40, 9-16.

(55) Koch, W.; Lutz, O.; Nolle, A.; ${ }^{77}$ Se and ${ }^{125}$ Te nuclear magnetic resonance investigations in II-VI and IV-VI compounds. Z. Phys. A: Hadrons and Nuclei 1978, 289, 17-20.

(56) Beckmann, P. A.; Schneider, E. Methyl group rotation, ${ }^{1} \mathrm{H}$ spin-lattice relaxation in an organic solid, and the analysis of non-exponential relaxation. J. Chem. Phys. 2012, 136, 054508.

(57) Panich, A. M.; Shao, M.; Teske, C. L.; Bensch, W. Size-dependent properties of $\mathrm{Tl}_{2} \mathrm{Se}$ studied by NMR spectroscopy. Phys. Rev. B 2006, 74, 233305.

(58) Hewes, C. R.; Adler, M. S.; Senturia, S. D. Annealing studies of PbTe and $\mathrm{Pb}_{1-\mathrm{x}} \mathrm{Sn}_{\mathrm{x}} \mathrm{Te} . J$. Appl. Phys. 1973, 44, $1327-1332$.

(59) Lim, P.N.; et. al, "Synthesis and Processing of nanostructured thermoelectric materials" SIMTech technical reports (STR_V12_N3_01_FTG) Volume 12 Number 3, 2011.

(60) Bouad, N.; Marin-Ayral, R.M.; Tedenac, J.C. Mechanical alloying and sintering of lead telluride. J. Alloys and Compounds 2000, 297(1-2), 312-318.

(61) Martin, J., Nolas, G.S.; Zhang, W.; Chen, L. PbTe nanocomposites synthesized from PbTe nanocrystals. Appl. Phys. Lett. 2007, 90, 222112-222112-3. 
(62) Oh, T.S.; Choi J.; Hyun, D.B. Formation of PbTe intermetallic compound by mechanical alloying of elemental $\mathrm{Pb}$ and Te powders. Scripta Metallurgica et Materialia 1995, 32(4), 595600.

(63) Bouad, N.; Marin-Ayral, R.M.; Tedenac, J.S. Mechanical alloying and sintering of lead telluride. J. Alloys and Compounds 2000, 297, 312-318.

(64) Lee, H.E.; Lee, D.Y.; Kim, I.J.; Woo, B.C. Manufacturing and characterization of nanosized PbTe powders. Thermoelectrics, Proceedings ICT '02. Twenty-First International Conference 2002.

(65) Gomez, M. P.; Stevenson, D. A.; Huggins, R. A. Self-Diffusion of Pb and Te in Lead Telluride. J. Phys. Chem. Solids 1971, 32, $335-344$.

(66) Yesinowski, J. P.; Ladouceur, H. D.; Purdy, A. P.; Miller, J. B. Electrical and ionic conductivity effects on magic-angle spinning nuclear magnetic resonance parameters of $\mathrm{CuI} . J$.

Chem. Phys. 2010, 133, 234509. 
Tables

Table I. Calculated Lead Chemical Shieldings for $\left[\mathrm{PbTe}_{6} \mathrm{H}_{6}\right]^{-4}$

\begin{tabular}{|c|c|c|c|c|c|}
\hline Pb-Te distance (pm) & $\sigma_{\text {dia }}(\mathbf{p p m})$ & $\begin{array}{l}\boldsymbol{\sigma}_{\text {para }} \\
(\mathbf{p p m})\end{array}$ & $\boldsymbol{\sigma}_{\text {So }}(\mathbf{p p m})$ & $\boldsymbol{\sigma}_{\text {iso }}(\mathbf{p p m})$ & $\boldsymbol{\delta}_{\text {iso }}(\mathbf{p p m})$ \\
\hline $\mathbf{2 9 8}$ & 9950 & -4960 & 2867 & 7857 & -513 \\
\hline $\mathbf{3 0 3}$ & 9950 & -5182 & 2830 & 7599 & -256 \\
\hline $\mathbf{3 0 8}$ & 9950 & -5463 & 2785 & 7273 & 71 \\
\hline $\mathbf{3 1 3}$ & 9950 & -5815 & 2734 & 6870 & 474 \\
\hline $\mathbf{3 1 8}$ & 9950 & -6249 & 2683 & 6384 & 959 \\
\hline $\mathbf{3 2 3}$ & 9950 & -6794 & 2636 & 5793 & 1550 \\
\hline $\mathbf{3 2 8}$ & 9950 & -7442 & 2606 & 5114 & 2229 \\
\hline
\end{tabular}


Table II. Calculated Tellurium Chemical Shieldings for $\left[\mathrm{TePb}_{6} \mathrm{~F}_{18}\right]^{-8}$

\begin{tabular}{|c|c|c|c|c|c|}
\hline Pb-Te distance (pm) & $\boldsymbol{\sigma}_{\text {dia }}(\mathbf{p p m})$ & $\begin{array}{c}\boldsymbol{\sigma}_{\text {para }} \\
\mathbf{p p m})\end{array}$ & $\boldsymbol{\sigma}_{\text {So }}(\mathbf{p p m})$ & $\boldsymbol{\sigma}_{\text {iso }}(\mathbf{p p m})$ & $\boldsymbol{\delta}_{\text {iso }}(\mathbf{p p m})$ \\
\hline $\mathbf{3 0 8}$ & 5311 & -2499 & 1357 & 4169 & -1070 \\
\hline $\mathbf{3 1 3}$ & 5311 & -2559 & 1439 & 4191 & -1093 \\
\hline $\mathbf{3 1 8}$ & 5311 & -2628 & 1529 & 4212 & -1113 \\
\hline $\mathbf{3 2 3}$ & 5311 & -2705 & 1626 & 4232 & -1134 \\
\hline $\mathbf{3 2 8}$ & 5311 & -2792 & 1733 & 4252 & -1153 \\
\hline $\mathbf{3 3 3}$ & 5311 & -2891 & 1846 & 4266 & -1167 \\
\hline
\end{tabular}


Table III. Comparison of $T_{1}$ relaxation times for lead telluride ball milled for various times. The average particle size of nanocrystalline powders was estimated from PXRD data using the Scherrer formula.

\begin{tabular}{|c|c|c|c|c|c|}
\hline vendor & $\begin{array}{l}\text { powdering } \\
\text { method }\end{array}$ & milling time & $\begin{array}{l}\text { average } \\
\text { nanocrystal } \\
\text { size }\end{array}$ & $T_{1}$ & $\begin{array}{l}\text { Kohlrausch } \\
\text { exponent ( } \beta \text { ) }\end{array}$ \\
\hline $\begin{array}{l}\text { Sigma- } \\
\text { Aldrich }\end{array}$ & $\begin{array}{l}\text { used as-is } \\
\text { (mesh140) }\end{array}$ & $\mathrm{n} / \mathrm{a}$ & $\mathrm{n} / \mathrm{a}$ & $2.6 \mathrm{~s}$ & 0.80 \\
\hline Alfa Aesar & mortar \& pestle & $\mathrm{n} / \mathrm{a}$ & $\mathrm{n} / \mathrm{a}$ & $6.8 \mathrm{~s}$ & 0.44 \\
\hline Alfa Aesar & ball milled & $1 \mathrm{~min}$. & $326 \mathrm{~nm}$ & $6.7 \mathrm{~s}$ & 0.53 \\
\hline Alfa Aesar & ball milled & $5 \mathrm{~min}$. & $151 \mathrm{~nm}$ & $12.7 \mathrm{~s}$ & 0.55 \\
\hline Alfa Aesar & ball milled & $15 \mathrm{~min}$. & $131 \mathrm{~nm}$ & $12.7 \mathrm{~s}$ & 0.55 \\
\hline Alfa Aesar & ball milled & $30 \mathrm{~min}$. & $98 \mathrm{~nm}$ & $12.7 \mathrm{~s}$ & 0.55 \\
\hline Alfa Aesar & ball milled & $60 \mathrm{~min}$. & $83 \mathrm{~nm}$ & $12.7 \mathrm{~s}$ & 0.55 \\
\hline
\end{tabular}


Figures and Captions

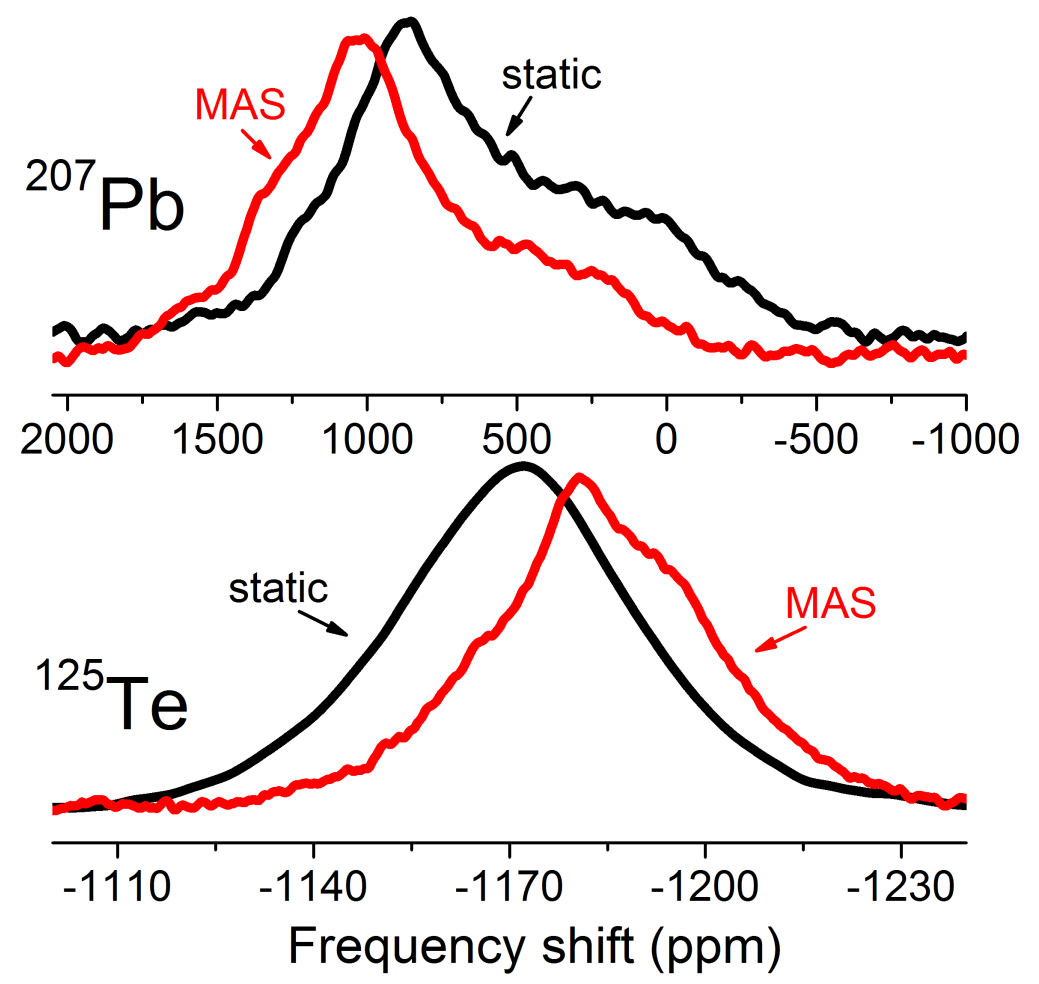

Figure 1. (a) ${ }^{207} \mathrm{~Pb}$ spectrum (black online, A) of static polycrystalline $\mathrm{PbTe}$ at $295 \mathrm{~K}$. The ${ }^{207} \mathrm{~Pb}$ MAS spectrum is shown below (red online, B). (b) ${ }^{125}$ Te spectrum (black online, A) of static polycrystalline PbTe at $295 \mathrm{~K}$. The ${ }^{125}$ Te MAS spectrum is shown below (red online, B). 


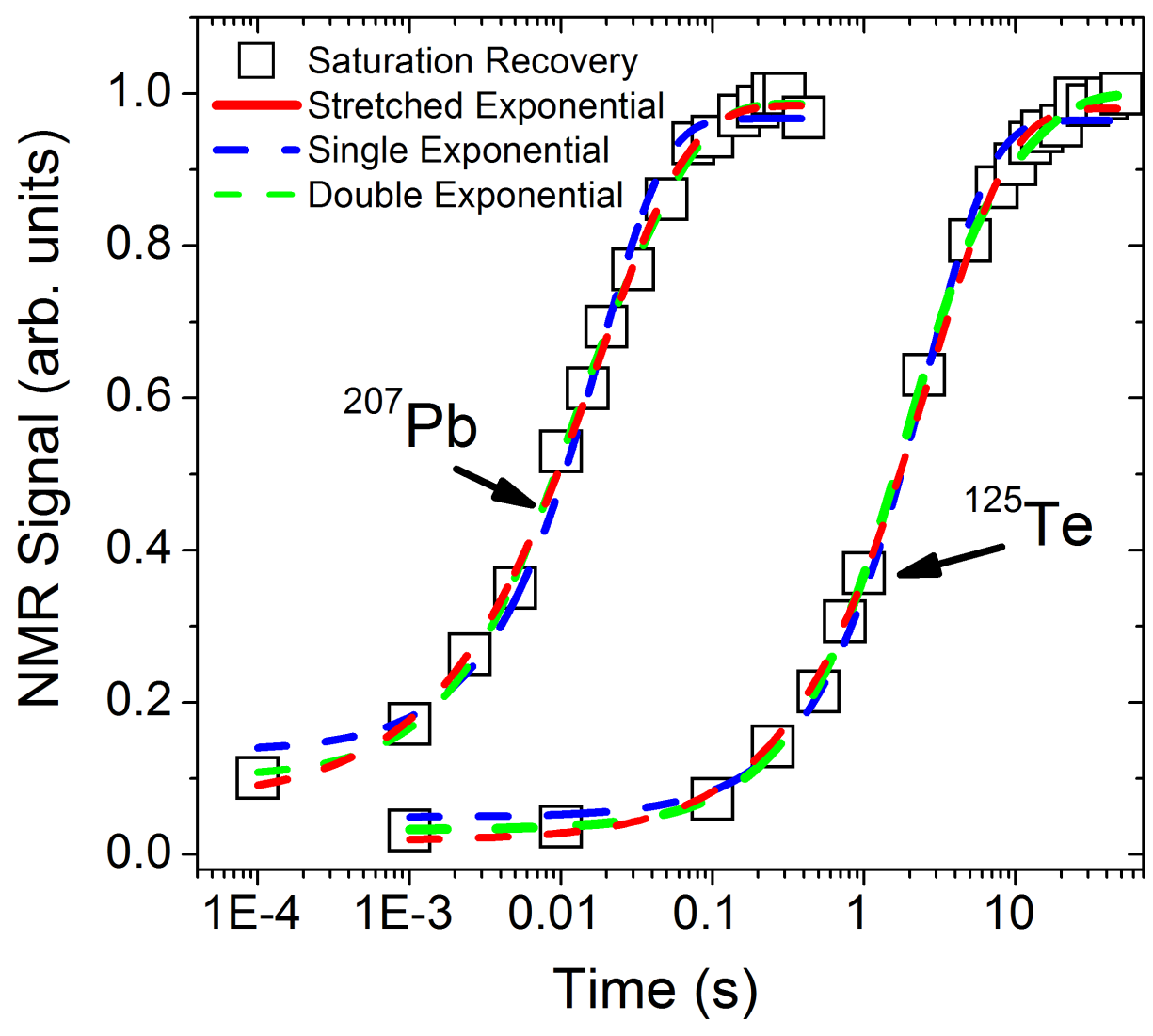

Figure 2. ${ }^{207} \mathrm{~Pb}$ and ${ }^{125} \mathrm{Te}$ saturation recovery of $\mathrm{PbTe}$. The data represent integral intensities of the entire resonance. The dashed line is a fit to a single-exponential (blue) and to a double exponential saturation-recovery model (green). The red line is a fit to a stretched-exponential saturation-recovery model. 


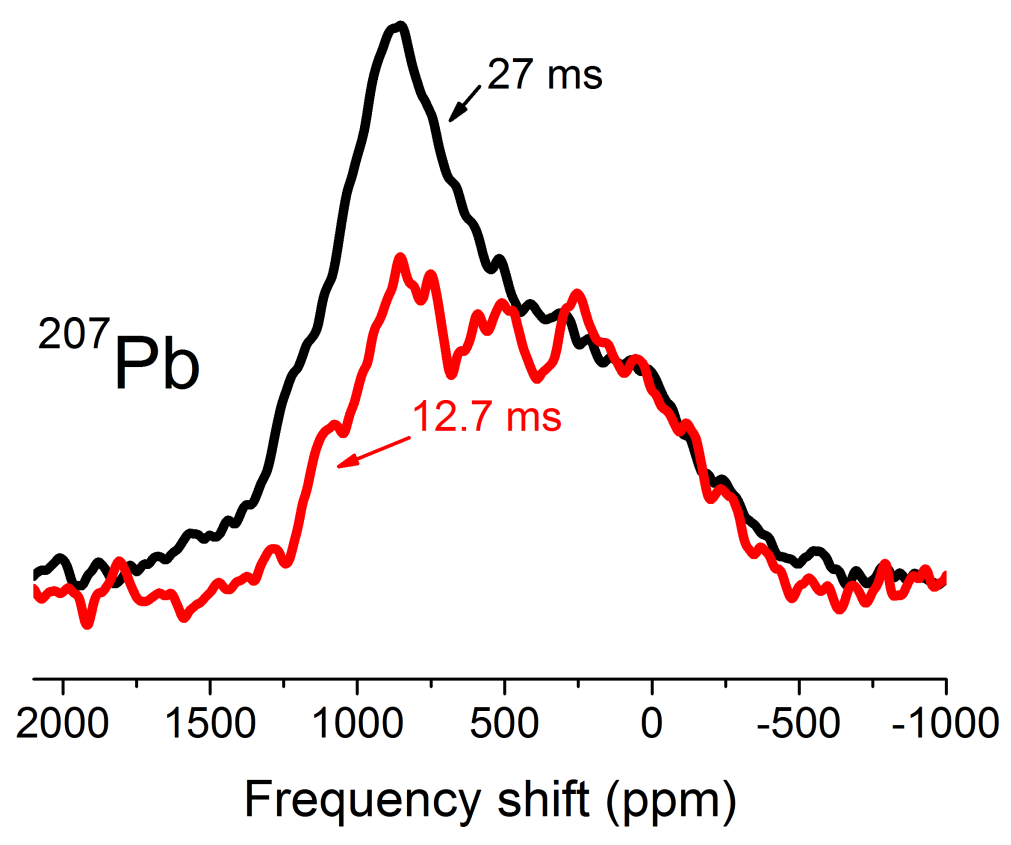

Figure 3. Comparison of the ${ }^{207} \mathrm{~Pb}$ spectrum obtained in the saturation-recovery experiment with a delay after saturation of $10 \mathrm{~ms}$ (red on line) and for a fully relaxed spectrum (black on line) of $\mathrm{PbTe}$ at $295 \mathrm{~K}$. Relaxation recovery is not uniform across the resonance. The regions at more negative shifts relax more quickly $(12.7 \mathrm{~ms})$ than do those at more positive shifts $(27 \mathrm{~ms}$, fully relaxed). 


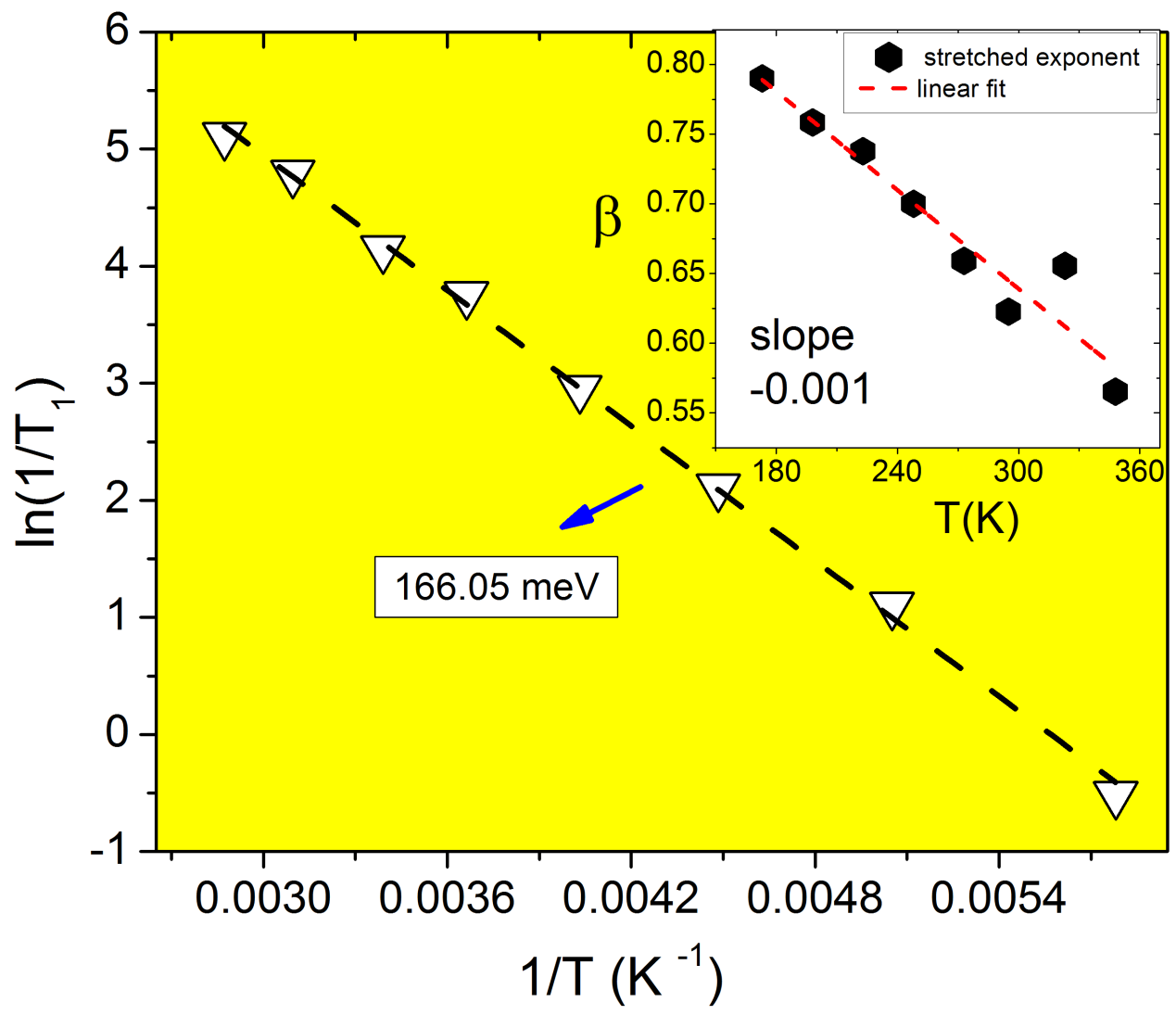

Figure 4. (a) The natural logarithm of the average ${ }^{207} \mathrm{~Pb}$ spin-lattice relaxation rate, as a function of the inverse temperature; (b) the exponent $(\beta)$ obtained from a stretched-exponential fit plotted as a linear function of the temperature. 


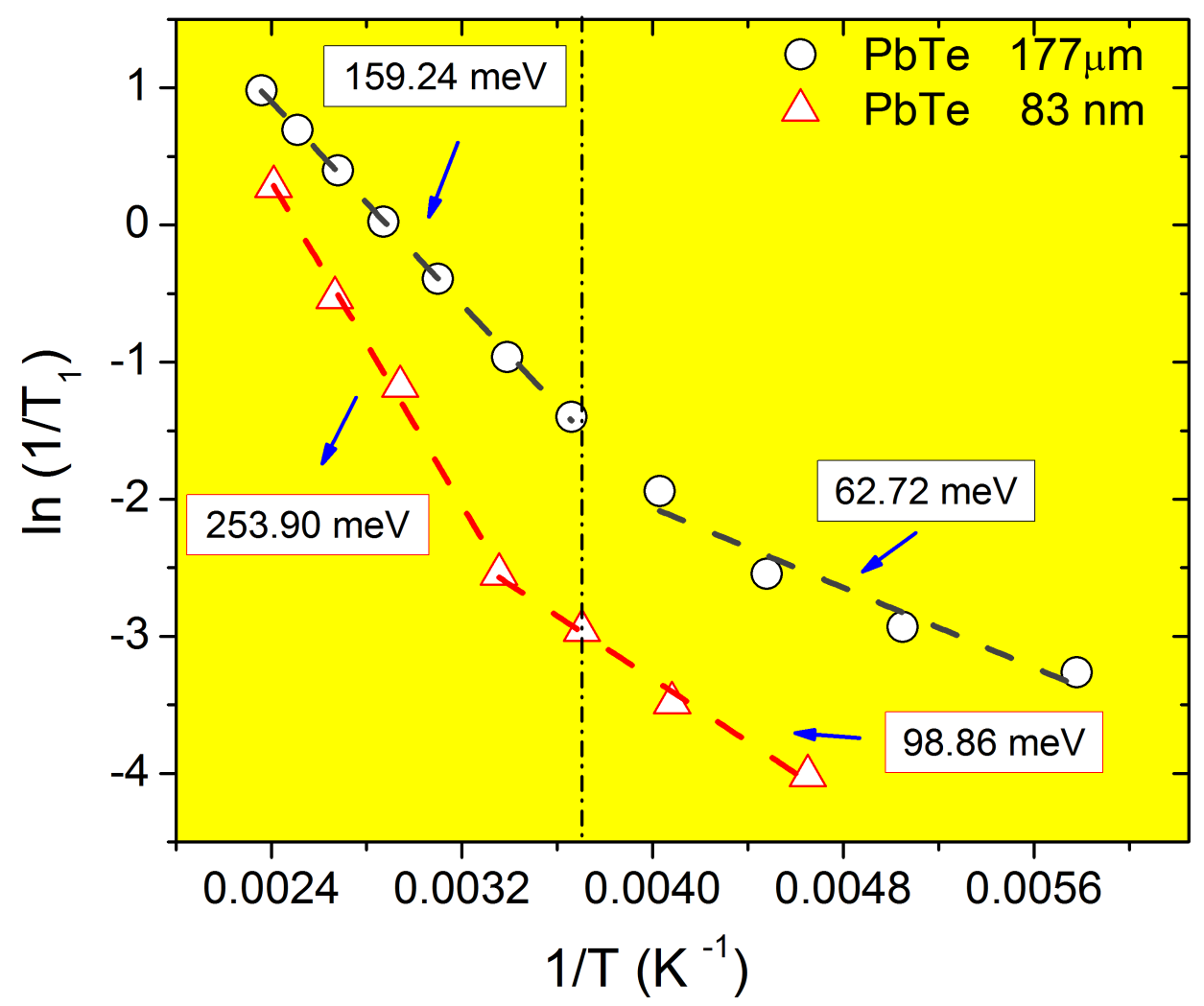

Figure 5. The natural logarithm of the ${ }^{125} \mathrm{Te}$ spin-lattice relaxation rate for the case of $\mathrm{PbTe}(177$ $\mu \mathrm{m}$, black open circles) and $\mathrm{PbTe}(83 \mathrm{~nm}$, red open triangles), as a function of the inverse temperature. Below $250 \mathrm{~K}$, a second mechanism dominates the spin lattice relaxation in both cases. 


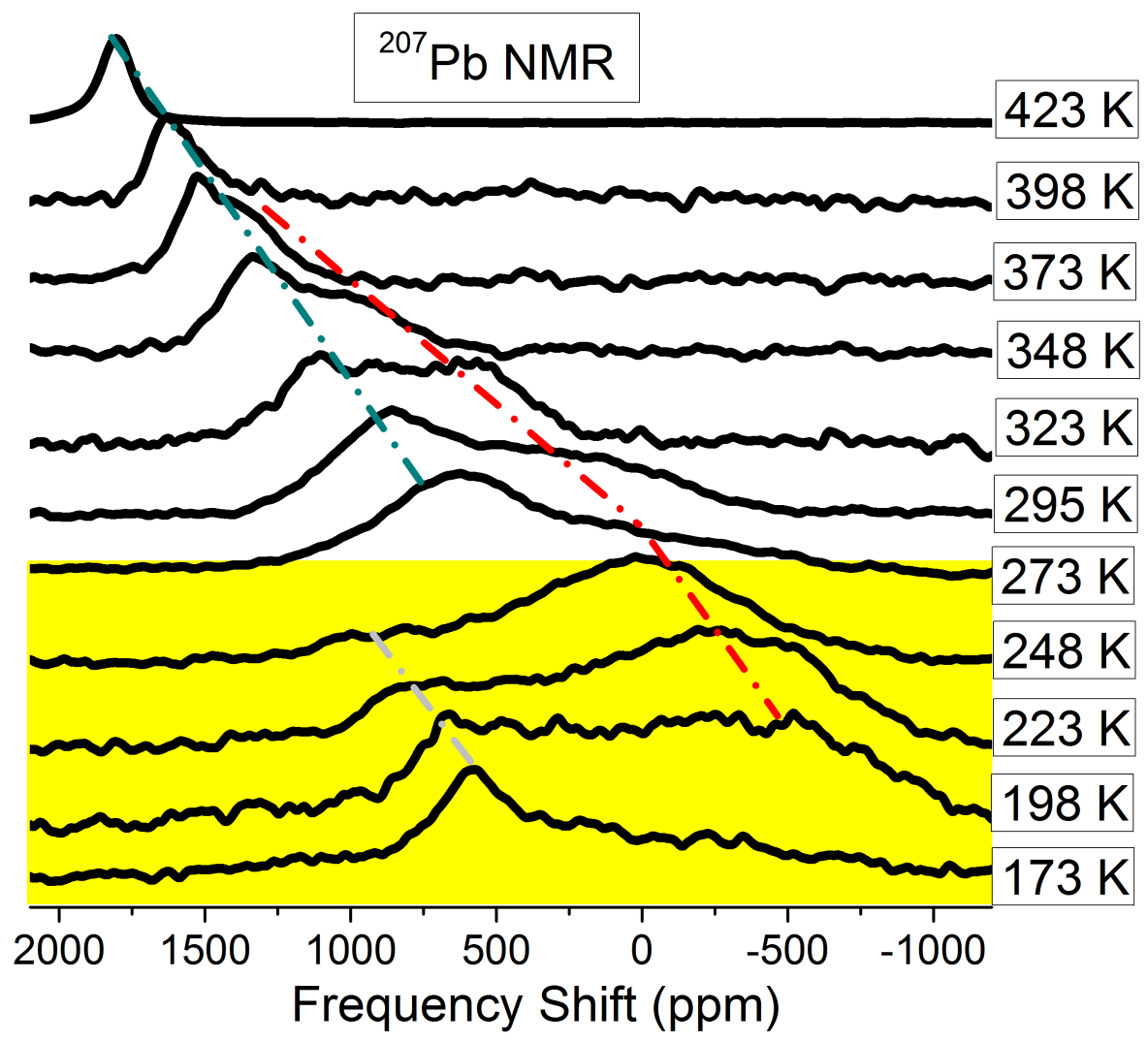

Figure 6. ${ }^{207} \mathrm{~Pb}$ NMR spectra of static polycrystalline PbTe from $173 \mathrm{~K}$ (bottom) to $423 \mathrm{~K}$ (top). The spectrum shape is changing as the temperature increases. Regions with different carrier concentrations shift differently with temperature (as shown by dashed lines). Above $250 \mathrm{~K}$ (white regime) the spectrum shifts to higher frequencies accompanied by a reduction in the spread of the spectrum. These two characteristics suggest an annealing of the native defects as the temperature increases. 


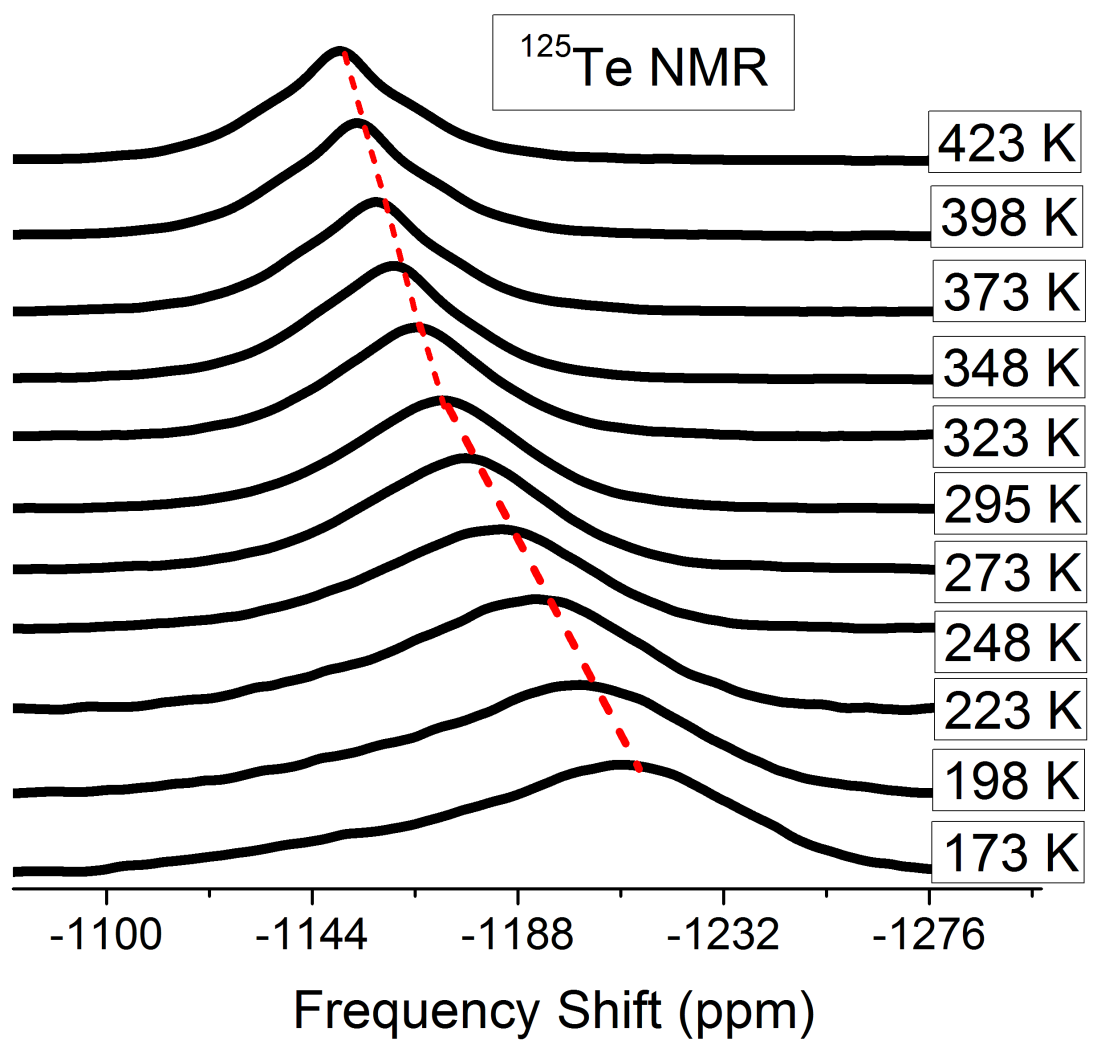

Figure 7. ${ }^{125}$ Te spectra of static polycrystalline PbTe from $173 \mathrm{~K}$ (bottom) to $423 \mathrm{~K}$ (top). With increasing temperature the observed positive shift of spectra is accompanied by a narrowing of the linewidth and by an alteration of ${ }^{125}$ Te spin-lattice relaxation mechanism. 


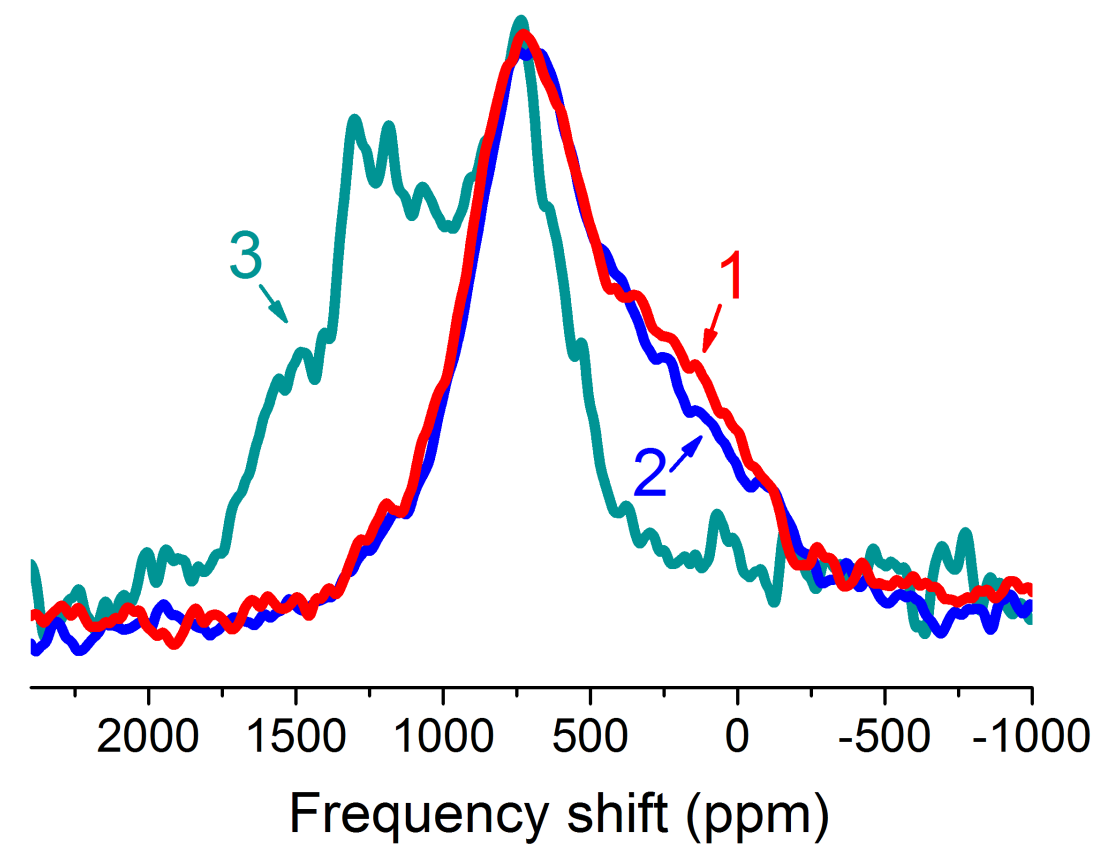

Figure 8. ${ }^{207} \mathrm{~Pb}$ spectra of static polycrystalline $\mathrm{PbTe}$ acquired at ambient temperature. The spectrum 1 (red on line, 1) is prior to any variable temperature experiments. The next spectrum (blue on line, 2) is at $295 \mathrm{~K}$ after acquiring data at $273 \mathrm{~K}$ and then up to and including $398 \mathrm{~K}$. The spectrum 3 (dark cyan on line, 3) was acquired at $295 \mathrm{~K}$ after first heating the sample to 423 $\mathrm{K}$. No further changes in the ambient temperature ${ }^{207} \mathrm{~Pb}$ spectrum were observed after the saturation-recovery experiments at any of the other lower temperatures. 


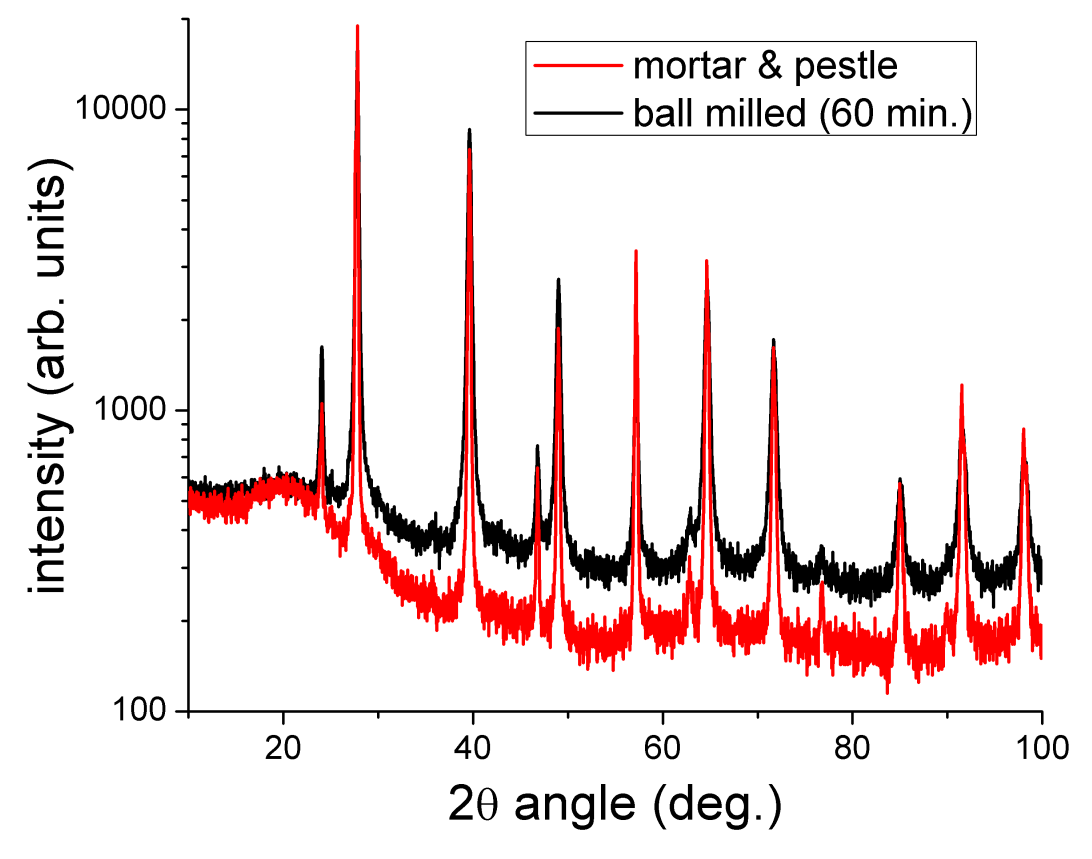

Figure 9. PXRD spectra for PbTe powders from mortar and pestle versus ball milled processes confirm that the samples preserve their crystallinity. For the ball-milled $(60 \mathrm{~min}$.) sample, the average particle size was estimated from the PXRD data to be $83 \mathrm{~nm}$, using the Scherer formula. 